\title{
A novel method to reconstruct phylogeny tree based on the chaos game representation
}

\author{
Na-Na Li ${ }^{1}$, Feng Shi ${ }^{1}$, Xiao-Hui Niu ${ }^{1,2^{*}}$, Jing-Bo Xia ${ }^{1}$ \\ ${ }^{1}$ College of Science, Huazhong Agricultural University, Wuhan, Hubei, China; \\ ${ }^{2}$ Tongji Medical College, School of Public Health, Wuhan, China. \\ Email: niuxiaoh@126.com
}

Received 5 September 2009; revised 20 September 2009; accepted 23 September 2009.

\begin{abstract}
We developed a new approach for the reconstruction of phylogeny trees based on the chaos game representation (CGR) of biological sequences. The chaos game representation (CGR) method generates a picture from a biological sequence, which displays both local and global patterns. The quantitative index of the biological sequence is extracted from the picture. The Kullback-Leibler discrimination information is used as a diversity indicator to measure the dissimilarity of each pair of biological sequences. The new method is inspected by two data sets: the Eutherian orders using concatenated $\mathrm{H}$-stranded amino acid sequences and the genome sequence of the SARS and coronavirus. The phylogeny trees constructed by the new method are consistent with the commonly accepted ones. These results are very promising and suggest more efforts for further developments.
\end{abstract}

Keywords: CGR (Chaos Game Representation); Discrimination Information; Phylogeny Tree

\section{INTRODUCTION}

Development of the nucleotide and protein sequencing technology have resulted in an explosive growth in the number of known DNA and protein sequences, it has raised many fundamental and challenging questions to modern biology. By analyzing a set of amino acid sequences (or proteins) of different species, reconstruction of the evolutionary history of genes and species is one of the most important subjects in the current study of molecular evolution. Although it is an important problem in bioinformatics, and, like many other problems, it is still an open subject for research. It is mainly due to the high degree of complexity of the problem [1] that leads to intractable search spaces when dealing with the phylogeny of a large number of species.

Current methods for the reconstruction of phylogeny trees can be roughly grouped into three kinds: maximum likelihood [2,3], maximum parsimony method [4] and distance-based methods. Maximum parsimony and maximum likelihood methods use previously aligned sequences of nucleotides as input, and they are less susceptible to errors. On the other hand, distance-based methods, such as UPGMA (unweighted pair group method using arithmetic averages) [5], Fitch-Margoliash [6] and neighbor-joining [7] use a matrix representing the distances between pairs of species, and they are based on the principle of similarity.

CGR [8,9] of biological sequences can investigate different hiding patterns of different biological sequences. It has been reported that for biological sequences at least 2000 bases are required to generate identifiable patterns [9], which do not depend on the order in which they are concatenated. In this paper, when the length of sequence is shorter than 2000 bases, we concatenate the sequence with itself repeatedly until the whole length has surpassed 2000. And we use the Kullback-Leibler discrimination information to measure the dissimilarity of each pair of biological sequences. The results proved the method is promising.

\section{MATERIALS AND METHODS}

\subsection{Data Sets}

In order to test our method, we have selected two test data, protein sequence and DNA sequence separately. The reconstruction of whole protein and nucleotide phylogenies using our new distance, all achieved very encouraging results.

\subsubsection{Protein Data Set}

It has been debated which two of three main groups of placental mammals are more closed related: Primates, Ferungulates, and Rodents. This is because by the maximum likelihood method, some proteins support the (Ferungulates, (primates, Rodents)) grouping while other proteins support the (Rodents, (Ferungulates, Primates)) grouping [10]. Cao et al. aligned 12 concatenated mito- 
chondrial proteins from the following species (available in the EMBL database (release 61)): human (Homo sapiens, V00622), common chimpanzee (Pan troglodytes, D38116), pygmy chimpanzee (Pan paniscus, D38113), gorilla (Gorilla gorilla, D38114), orangutan (pongo pygmaeus, D38115), gibbon (Hylobates lar, X99256), Sumatran orangutan (pongo pygmaeus abelii, X97707), rat (Rattus norvegicus, X14848), house mouse (Mus musculus, V00711), grey seal (Halichoerus grypus, X72004), harbor seal (Phoca vitulina, X63726), cat (Felis catus, U20753), white rhino (Ceratotherium simum, Y07726), horse (Equus caballus, X79547), finback whale (Balaenoptera physalus, X61145), blue whale (Balaenoptera musculus, X72204), cow (Bos taurus, V00654), using opossum (Didelphis virginiana, Z29573), wallaroo (Macropus robustus, Y10524) and platypus (Ornithorhynchus anatinus, X83427) as the out-group, and built the maximum likelihood tree to confirm the grouping (Rodents, (Primates, Ferungulates)). So we select this controversial data set to test our method.

\subsubsection{DNA Data Set}

From NCBI (National center for biotechnology information), we download the 12 coronavirus sequences and 12 SARS virus sequences $[11,12]$ that have been cultured isolating from the index case from all over the world. The 24 complete genome sequences' logo, accession, host, and location are listed in the Table 1.

\subsection{Chaos Game of Representation of Proteins}

It is known that the protein sequence is formed by 20 different kinds of amino acids. Basu. et al. [8] classify 20 kinds of amino acids to 12 different groups according to

Table 1. Coronaviruses and SARS virus sequences' information.

\begin{tabular}{|c|c|c|c|}
\hline Logo & Accession & Host & Location \\
\hline cAvian & NC_001451.1 & Avian & \\
\hline cBovine_1 & AF391541.1 & Bovine & \\
\hline cBovine_2 & AF391542.1 & Bovine & \\
\hline cBovine_3 & U00735.2 & Bovine & \\
\hline cBovine_4 & AF220295.1 & Bovine & \\
\hline cHuman & AF304460.1 & Human & \\
\hline cMouse & AF029248.1 & Murine & \\
\hline cMurine_1 & AF208066.1 & Murine & \\
\hline cMurine_2 & AF201929.1 & Murine & \\
\hline cMurine_- 3 & AF208067.1 & Murine & \\
\hline cPig_1 & NC_002306.2 & Pig & \\
\hline cPig_2 & $\mathrm{NC}_{-}^{-} 003436.1$ & Pig & \\
\hline SARS_BJ01 & AY 278488.2 & Human & Beijing \\
\hline SARS_HK_1 & AY282752.1 & Human & Hong Kong \\
\hline SARS_HK_2 & AY278491.2 & Human & Hong Kong \\
\hline SARS_HK_3 & AY278554.2 & Human & Hong Kong \\
\hline SARS_SG_1 & AY283794.1 & Human & Singapore \\
\hline SARS_SG_2 & AY283795.1 & Human & Singapore \\
\hline SARS_SG_3 & AY283796.1 & Human & Singapore \\
\hline SARS_SG_4 & AY283797.1 & Human & Singapore \\
\hline SARS_SG_5 & AY283798.1 & Human & Singapore \\
\hline SARS_TOR2 & AY274119.3 & Human & Toronto in Canada \\
\hline SARS_TW1 & AY291451.1 & Human & Taiwan \\
\hline SARS_Urban & AY278741.1 & Human & United States \\
\hline
\end{tabular}

their different conservative substitutions such as alanine (A) and glycine $(\mathrm{G})$, are considered as one vertex; serine (S) and threonine (T) represent a vertex; and so on. Furthermore, Basu. et al. claims that the following 12-vertex CGR algorithm is optimum for generation of distinct patterns for different protein families.

Following the chaos game algorithm, the first amino acid residue of the concatenated protein sequence is plotted halfway between the random initial point and the vertex labelled with the first residue. The second residue in the sequence is then plotted halfway between the first point and the vertex labelled with the second residue. The process must be repeated until the last residue in the sequence is plotted.

The 12-sided polygon is divided into 24 segments (grid) as shown in Figure 1 and the segments are labelled serially with numbers 1-24. For each segment, says $S_{k}$, we count the number of points fall in $S_{k}$, says $L_{k}$. (The points falling on boundaries should be counted in any one of the neighboring segments). Then set $\mathrm{G}_{\mathrm{k}}=$ $\mathrm{L}_{\mathrm{k}} / \mathrm{N} ; \mathrm{k}=1 ; 2 ; \ldots ; 24$; where $\mathrm{N}$ is the length of the protein sequence. From the above 12-vertex CGR algorithm, we can transform each protein sequence into a 24-dimensional vector $\left(\mathrm{G}_{1} ; \ldots ; \mathrm{G}_{24}\right)$.

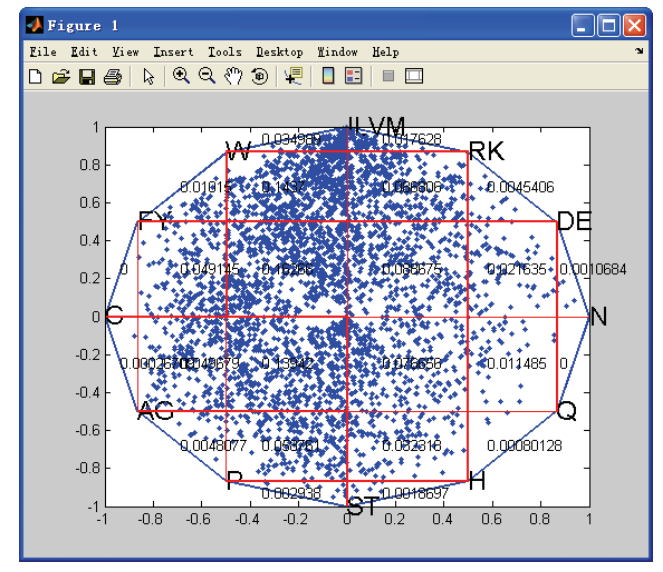

Figure 1. Chaos game representation of protein.

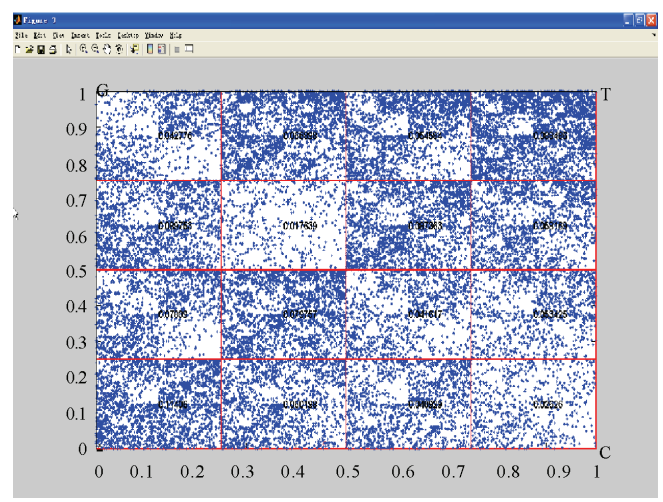

Figure 2. Chaos game of representation of DNA. 


\subsection{Chaos Game Representation of DNA Sequence}

Similar to the chaos game representation of proteins, each of the four vertex of the square is labelled ' $a$ ', ' $c$ ', ' $\mathrm{g}$ ', or ' $\mathrm{u}$ '. According to the DNA sequence [9], we plot half way between the random initial point and the vertex labeled with the first nucleotide acid. Then the second nucleotide acid in the sequence is plotted halfway between the first point and the vertex labelled with the second one. Following this method, it is repeated until the last nucleotide acid is plotted.

The square is divided into 16 segments (shown in Figure 2). Each of segments is labelled with the numbers 1-16.Then we can count the percent of the points that are fallen into each of segment. Following this algorithm, each DNA sequence will induce a 16-dimensional vector $(\mathrm{G} 1 ; \ldots ; \mathrm{G} 16)$.

\subsection{The Kullback-Leibler Discrimination Information}

$\mathrm{X}$ is a discrete random variable. It has the different distribution laws under the different hypotheses. Such as, under hypothesis H1, its distribution law is defined as follow:

$$
\left(\begin{array}{c}
X \\
p_{1}(x)
\end{array}\right)=\left(\begin{array}{cccc}
a_{1} & a_{2} & \cdots & a_{K} \\
p_{1}\left(a_{1}\right) & p_{1}\left(a_{2}\right) & \cdots & p_{1}\left(a_{K}\right)
\end{array}\right)
$$

By similarity, under hypothesis $\mathrm{H} 2$, its distribution is similar:

$$
\left(\begin{array}{c}
X \\
p_{2}(x)
\end{array}\right)=\left(\begin{array}{cccc}
a_{1} & a_{2} & \cdots & a_{K} \\
p_{2}\left(a_{1}\right) & p_{2}\left(a_{2}\right) & \cdots & p_{2}\left(a_{K}\right)
\end{array}\right)
$$

The Kullback-Leibler discrimination information between the two distributions is defined as follow:

$$
I\left(p_{1}, p_{2}\right)=\sum_{i=1}^{K} p_{1}\left(a_{i}\right) \log \frac{p_{1}\left(a_{i}\right)}{p_{2}\left(a_{i}\right)}
$$

The detailed step to measure the dissimilarity using this concept is listed as follow.

For example, there are two sequences, $\mathrm{X}$ and Y. Following the CGR algorithm, they can transform into the vector of the percent, $\left(\mathrm{G}_{\mathrm{X}}(1) ; \ldots ; \mathrm{G}_{\mathrm{X}}(\mathrm{k})\right)$ and $\left(\mathrm{G}_{\mathrm{Y}}(1) ; \ldots\right.$; $\left.\mathrm{G}_{\mathrm{Y}}(\mathrm{k})\right) \mathrm{k}=16$ or 24 , according to the kind of biological sequence. The two vectors can be seen as the two different distribution laws.

$$
I(X, Y)=\sum_{i=1}^{k} G_{X}(i) \log \frac{G_{X}(i)}{G_{Y}(i)} \lim _{x \rightarrow \infty}
$$

Then the Kullback-Leibler discrimination information of two frequencies distribution is defined as follow:

$\mathrm{I}(\mathrm{X}, \mathrm{Y})$ denote the discrimination information between the $\mathrm{X}$ and $\mathrm{Y}$. It is should be noted that maybe some $\mathrm{G}_{\mathrm{Y}}(\mathrm{i})$ $=0$, this make $G_{X}(i) / G_{Y}(i)$ no sense. In this case, we may treat $\mathrm{G}_{\mathrm{Y}}(\mathrm{i})$ as a very small positive real number, and this would not cause trouble, and make our discussion very conversional. At the same, we always note that $0 \cdot \log 0=0$.

Because the discrimination information has direction (also termed as directed divergence), it is $\mathrm{I}(\mathrm{X}, \mathrm{Y}) \neq \mathrm{I}(\mathrm{Y}$, $\mathrm{X})$ in general, so we now introduce another measure $\mathrm{J}(\mathrm{X}$, $\mathrm{Y})$ as the following:

$$
\mathrm{J}(\mathrm{X}, \mathrm{Y})=\mathrm{I}(\mathrm{X}, \mathrm{Y})+\mathrm{I}(\mathrm{Y}, \mathrm{X})
$$

Then $\mathrm{J}(\mathrm{X}, \mathrm{Y})$ has the following properties:

(1) $\mathrm{J}(\mathrm{X}, \mathrm{Y}) \geq 0$

(2) $\mathrm{J}(\mathrm{X}, \mathrm{Y})=0$ if and only if $\mathrm{X}=\mathrm{Y}$.

(3) $\mathrm{J}(\mathrm{X}, \mathrm{Y})=\mathrm{J}(\mathrm{Y}, \mathrm{X})$.

At last, we introduce Distance $(\mathrm{X}, \mathrm{Y})$ to measure the diversity (dissimilarity) of the biological sequences, $\mathrm{X}$ and $\mathrm{Y}$.

\section{RESULTS}

\subsection{Protein Data Set}

With the protein data set, firstly, the out-group species separate from other mammals. Secondly, the three classes grouped each other obviously. Above all, we computed the Distance (X, Y) for each pair of species X and $\mathrm{Y}$ and constructed a tree (shown in Figure 3) using the neighbor joining [7] program in the MOLPHY package. The tree is very close to the maximum likelihood tree of Cao et al [10]. We also support the collusion of the (Rodents, (Ferungulates, Primates)) grouping. And we try to connect the midpoint of every edge to divide the polygon into 84 segments. Then following the same routine, we get the similar phylogeny tree, there is one difference from the previous tree that the horse's position is different.

\subsection{DNA Data Set}

With the DNA data set, we reconstructed the phylogeny tree (shown in Figure 4), separated the coronavirus sequences and SARS sequences completely. And the SARS sequences are more resemble to the first group of coronavirus. These results are similar to the commonly accepted results [13]. The 12 SARS virus sequences are obviously separated from the 12 coronavirus sequences. It supports the conclusion that SARS virus belong to the coronavirus, but they are different from the conventional coronavirus. On the phylogeny tree, SARS viruses are closest to the c_pig1, c_pig2 and c_Human which belong to the first kind of the coronavirus according to the serotype. It shows that SARS virus is nearest to the first kind of coronavirus. This is different from the Rota et al [13]. But it supports the experiment result of the Ksiazek et al [14].

Then we further divide the every segment into four average parts. That is to say, we divide the square into 64 segments. With the same method, we get the completely same tree. 


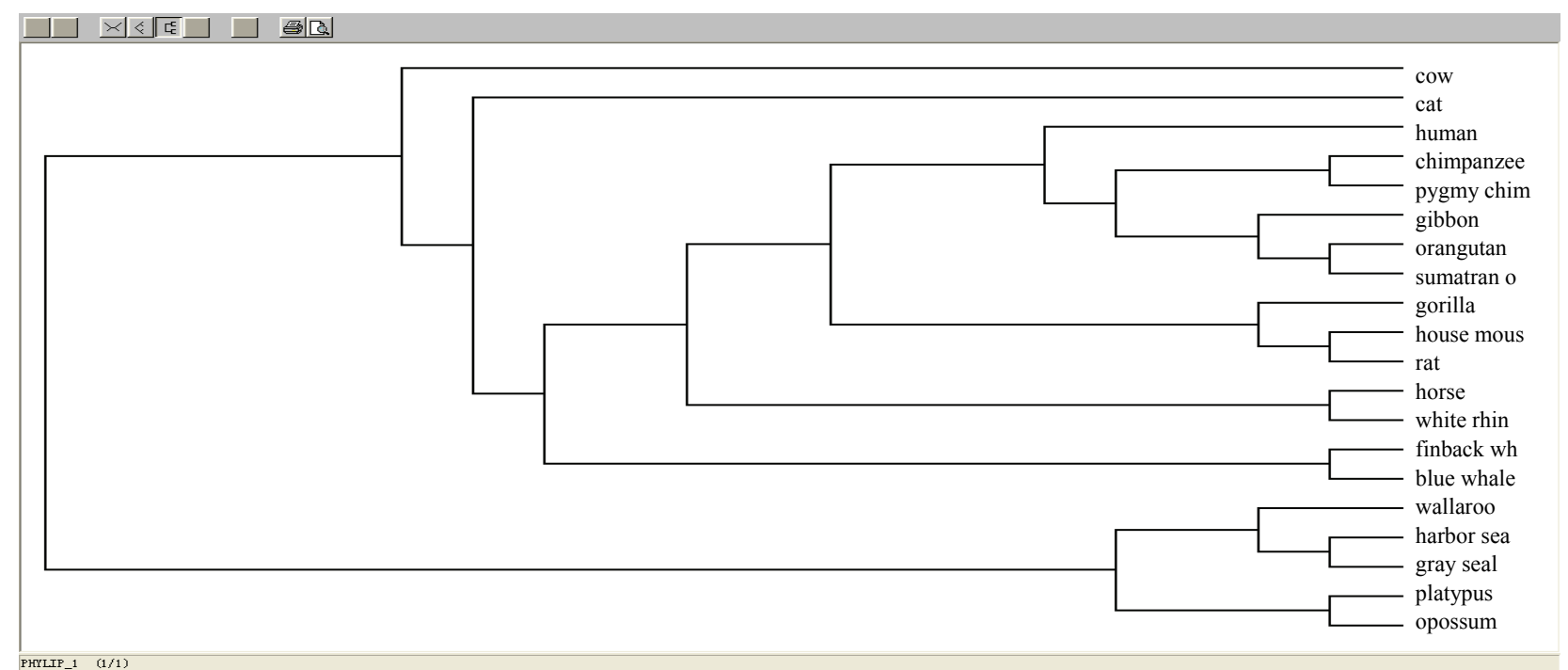

Figure 3. Phylogeny tree with the mitochondrial proteins from 20 species.

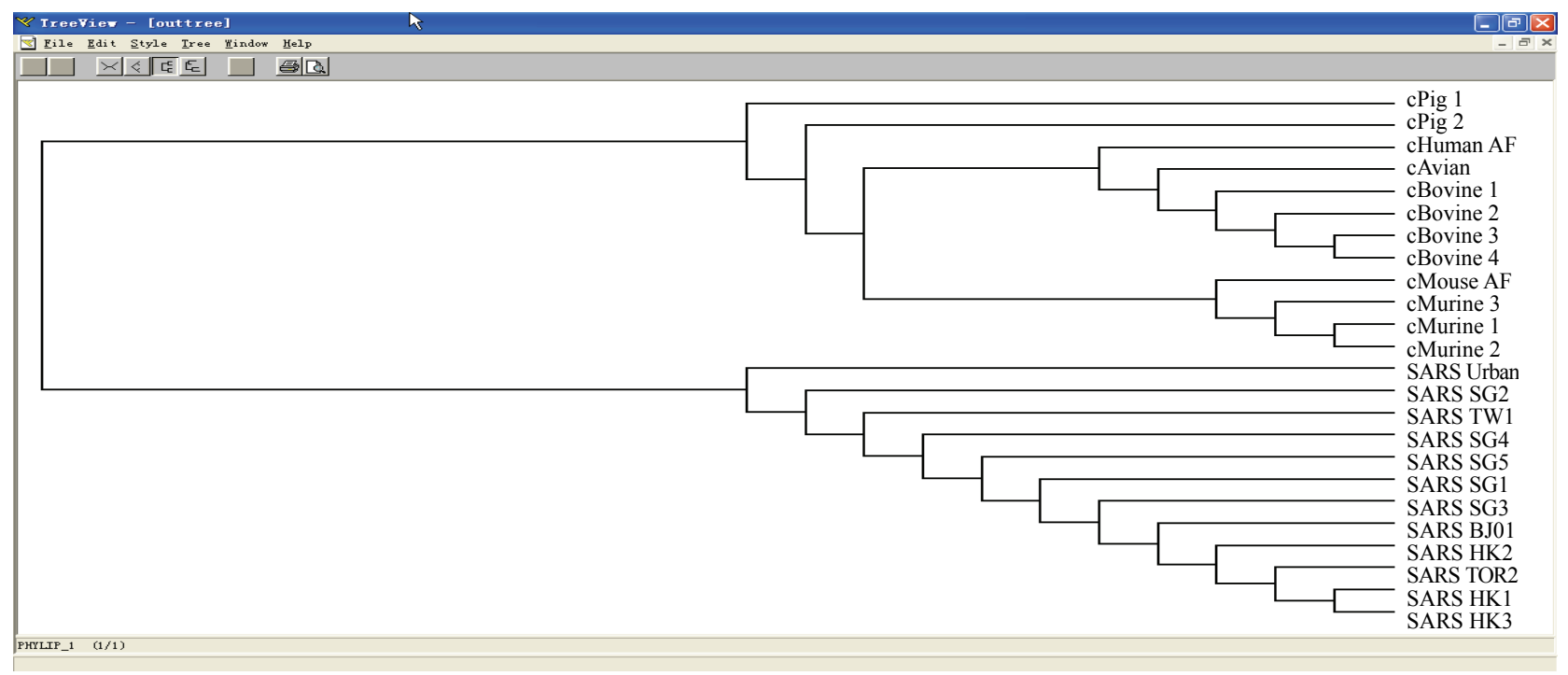

Figure 4. Phylogeny tree with coronavirus and SARS virus sequences.

\section{CONCLUSIONS}

We develop the new method based on the CGR of biological sequences. We achieved the promising results. This method is universal. It can reconstruct the phylogeny tree not only with the protein sequences data but also with the DNA (or RNA) sequences data. The numerical experiments show its stability. We tried to divide the square (or polygon) into more segments, and then we reconstruct the phylogeny tree in the similar way. We achieved the similar results. That is to say, the CGR method can show the distinct pattern for different proteins, no matter how to divide the pictures. And the Kullback-Leibler discrimination information can measure the dissimilarity of the proteins rightly.

The successful application to reconstruct the phylog- eny tree means that this new measurement of the dissimilarity between the biological molecules can not only use to reconstruct the phylogeny tree, but also apply to other comparative genomics research communities.

\section{REFERENCES}

[1] G. H. Gonnet. (1994) New algorithms for the computation of evolutionary phylogenetic trees [M], ComputationalMethods in Genome Research (Suhai, S., ed.), Plenum, New York, 153-161.

[2] L. L. Cavalli Sforza and A. W. Edwards. (1967) Phylogenetic analysis: Models and estimation procedures [J], Genetics, 19(3), 233-257.

[3] J. Felesenstein. (1981) Evolutionary trees from DNA sequences: A maximum likelihood approach [J], J Mol Evol, 17(6), 368-376. 
[4] L. Jin and M. Nei. (1990) Limitation of the evolution parsimony method of phylogenetic analysis [J], Mol Biol Evol, 7(1), 82-102.

[5] R. R. Sokal and C. D. Michener. (1958). A statistical method for evaluating systematic relationships [J], Univ. Kans. Sci. Bull, 28, 1409-1438.

[6] Chris. (2004) Fitch-Margoliash algorithm for calculating the branch lengths [EB/OL],

http://www.bioinfo.rpi.edu/ bystrc/courses/biol4540/lectur e12/sld002.htm.

[7] N. Saitou and M. Nei. (1987) The neighbor-joining method: A new method for reconstructing phylogenetic trees [J], Molecular Biology and Evolution, 4(4), 406425 .

[8] S. Basu, A. Pan, C. Dutta and J. Das. (1997) Chaos game representation of protein, J. Mol. Graphics Model, 15, 279-289.

[9] H. J. Jeffrey. (1990) Chaos game representation of gene structure [J], Nucleic AcidsRes., 18, 2163-2170.
[10] Y. Cao, N. Okada, and M. Hasegawa. (1997) Phylogenetuc position of guinea pigs revisited [J], Mol. Biol. Evol., 14, 461-464.

[11] M. A. Marra, S. J. Jones, C. R. Astell, et al. (2003) The genome sequence of the SARS-associated coronavirus [J], Science, 300(5624), 1399-1404.

[12] Y. J. Ruan, C. L. Wei, L. A. Ee, et al. (2003) Comparative full-length genome sequence analysis of 14 SARS coronavirus isolates and common mutations associated with putative origins of infection [J], The Lancet, 361(9371), 1779-1785.

[13] P. A. Rota, M. S. Oberste, S. S. Monroe, et al. (2003) Characterization of a novel coronavirus associated with severe acute respiratory syndrome $[\mathrm{J}]$, Science, $\mathbf{3 0 0}$ (5624), 1394-1399.

[14] T. G. Ksiazek, D. Erdman, C. Goldsmith, et al. (2003) A novel coronavirus associated with severe acute respiratory syndrome [J], N Engl J Med, 348(20), 1953-1966. 